GLOBAL SCIENCE AND TECHNOLOGY (ISSN 1984 - 3801)

\title{
GERMINAÇÃO DE FRUTOS VERDES E MADUROS DE Syagrus oleracea Becc. EM FUNÇÃO DO TAMANHO
}

\author{
Núbia Sousa Carrijo $^{1 *}$, Edésio Fialho dos Reis ${ }^{2}$, Antônio Paulino da Costa Netto ${ }^{3}$
}

\begin{abstract}
RESUMO: O presente trabalho teve como objetivo verificar se o tamanho do fruto afeta a germinação de sementes provenientes de frutos verdes e maduros de Syagrus oleracea Becc. (Arecaceae), conhecida popularmente como guariroba. Para tanto, foram coletados frutos de guariroba nos municípios de Mineiros e Jataí (GO), mensurados e separados em cinco classes de tamanho (T1: fruto $\leq 4,300 \mathrm{~cm} ; \mathrm{T} 2: 4,300 \mathrm{~cm}<$ fruto $\leq 4,833 \mathrm{~cm} ;$ T3: 4,833 cm < fruto $\leq 5,367 \mathrm{~cm} ; \mathrm{T} 4: 5,367 \mathrm{~cm}<$ fruto $\leq 5,900 \mathrm{~cm}$ e T5: fruto $>5,900$ $\mathrm{cm})$. Em câmaras de germinação tipo BOD, foram realizados ensaios com frutos verdes e maduros, nestes ensaios foram calculados índice de velocidade de germinação (IVG), porcentagem de germinação (\%G) e tempo gasto para germinar metade dos frutos $\left(\mathrm{T}_{50}\right)$. Foi ainda realizada análise de variância para as características avaliadas. Maiores $\% \mathrm{G}$ foram encontradas em frutos com comprimento entre 4,834 e 5,367 $\mathrm{cm}$ e menores $\% \mathrm{G}$ entre 5,368 e 5,90 cm. Frutos maduros possuem maior capacidade de germinação quando comparados com frutos verdes.
\end{abstract}

Palavras-chave: Tamanho de fruto, desenvolvimento inicial, germinação, guariroba.

\section{GERMINATION OF GREEN AND RIPE FRUITS OF Syagrus oleracea Becc. DEPENDING ON THE SIZE}

\begin{abstract}
This study aimed to verify if the size of the fruit affects the germination of seeds from green and ripe fruits of Syagrus oleracea Becc. (Arecaceae), known popularly as guariroba. To this end, we collected fruits guariroba in the municipalities of Mineiros and Jataí (GO), measured and separated in to five size classes (T1: fruit $\leq 4,300 \mathrm{~cm}$; T2: $4,300 \mathrm{~cm}<$ fruit $\leq 4,833 \mathrm{~cm}$; T3: $4,833 \mathrm{~cm}<$ fruit $\leq 5,367 \mathrm{~cm}$; T4: $5,367 \mathrm{~cm}<$ fruit $\leq 5,900 \mathrm{~cm}$ and T5: fruit $>$ $5,900 \mathrm{~cm}$ ). In germination chambers BOD tests were carried out with green and ripe fruits, these tests were calculated germination speed index(IVG), germination $(\% \mathrm{G})$ and time taken to germinate half of the fruits $\left(\mathrm{T}_{50}\right)$. It was also performed analysis of variance for the traits evaluated. In the laboratory, higher $\% \mathrm{G}$ were found in fruit length between 4,834 and $5,367 \mathrm{~cm}$ and smaller $\% \mathrm{G}$ between 5,368 and $5,90 \mathrm{~cm}$. Mature fruits have a higher germination when compared with green fruits.
\end{abstract}

Keywords: early development, fruit size, germination, guariroba.

\footnotetext{
${ }^{1}$ Universidade Estadual de Goiás, Unidade Mineiros. Av. 07, Quadra 02, Lote 01, Martins-Centro, Mineiros (GO). CEP: 75830-000. *E-mail: florestabuba@yahoo.com.br. Autor para correspondência.

${ }^{2}$ Universidade Federal de Goiás - UFG - Campus Jataí. Rua Riachuelo, 1530, Samuel Graham, Caixa Postal 03, Jataí (GO). CEP: 75804-020.

${ }^{3}$ Universidade Federal de Goiás - UFG - Campus Avançado de Jataí - CAJ. Unidade Jatobá, Rod. BR 364, km 192, n. 3800, Laboratório de Fisiologia Vegetal e Sementes, Parque Industrial, Caixa Postal 03, Jataí (GO). CEP: 75801-615.
}

Recebido em: 10/07/2012. Aprovado em: 10/04/2013. 


\section{INTRODUÇÃO}

Apesar de o Brasil ser um grande produtor, consumidor e exportador de palmito doce em conserva, ainda há poucas informações científicas referentes à produção de mudas e cultivo da guariroba [Syagrus oleracea Becc.], a qual é a única espécie produtora de palmito amargo (BATISTA, 2009).

A Syagrus oleracea Becc. é popularmente conhecida como gueroba, gueiroba, guariroba, gariroba. É comum nos campos sujos de cerrado, ocorrendo nos estados de São Paulo, Minas Gerais, Mato Grosso, Goiás, Mato Grosso do Sul, Bahia e Distrito Federal (SANTELLI et al., 2006).

Segundo Nascente (2009), a guariroba é uma palmeira que possui caule único, não perfilha e apresenta grande variabilidade quanto ao ponto de colheita, podendo ser colhida entre dois anos e meio até seis anos. Frutifica abundantemente na primavera, sendo que $1 \mathrm{~kg}$ de diásporos contém cerca de 70 unidades, os quais germinam em 2 a 3 meses (LORENZI et al., 2004).

A guariroba é uma palmeira nativa, importante no contexto do desenvolvimento regional pelos vários produtos que pode fornecer (SANTELLI et al., 2006).

O palmito amargo é amplamente empregado na culinária local, na confecção de pratos típicos ou apenas cozido, principalmente nos estados de Goiás e Minas Gerais (NASCENTE, 2009).

Segundo Lorenzi et al. (2004), o tamanho dos frutos de arecáceas possuem grande variação de tamanho. Ainda para os mesmos autores, o embrião e o albúmen são dissolvidos por um envoltório mais ou menos espesso, castanha, que os protege e cujo papel termina na germinação. A localização do embrião é indicada pela posição do opérculo.

Para Alves et al. (2005), a classificação das sementes por tamanho tem sido bastante utilizada para diferentes espécies, com a finalidade de determinação de fatores de qualidade fisiológica, através de testes de germinação e vigor.
No geral, as sementes grandes têm sido correlacionadas com maiores taxas de crescimento inicial de plântulas, o que pode ser um indício de sucesso durante o seu estabelecimento, já que o rápido crescimento de raiz e parte aérea permite à planta aproveitar as reservas nutricionais e hídricas do solo e realizar a fotossíntese (ALVES et al., 2005).

Os frutos devem ser colhidos no ponto de maturidade fisiológica, que é o momento em que suas sementes atingem a máxima qualidade fisiológica, apresentando a maior porcentagem de germinação e vigor. Carvalho e Nakagawa (2000) recomendaram que devessem ser considerados também, os aspectos relacionados com as características de natureza morfológica e física das sementes, como: dimensões, grau de umidade e conteúdo de matéria seca.

O estádio de maturação da semente é um fator que influencia na germinação. Para as palmeiras, conforme Lorenzi et al. (2004), as melhores porcentagens de germinação são obtidas a partir de frutos maduros, sendo muito falha quando obtidas a partir de frutos imaturos, pois o endosperma ainda encontrase aquoso, não solidificado. Entretanto, Viana (2003) estudando a espécie Livistona rotundifolia (Lam.) Mart., obteve melhores resultados com sementes de frutos colhidos verdes.

Dos estudos realizados sobre a germinação de sementes de palmeiras, pôdese observar que em sua maioria, utilizou-se em diferentes graus de desenvolvimento, tendo como parâmetro a cor dos frutos. Desse modo, o estudo de maturação de sementes com coletas periódicas nessas espécies é ainda raro e requer conhecimentos anteriores como condições ideais para germinação e, principalmente temperatura, substrato e fotoperíodo (IOSSI, 2005).

Conhecer o processo da germinação e dos aspectos morfológicos é importante para estudos taxonômicos, ecológicos e agronômicos. Na maioria das palmeiras, o processo germinativo ainda não foi completamente descrito, assim como a 
estrutura das plântulas em formação não foram identificadas (GENTIL \& FERREIRA, 2005).

A propagação da maioria das palmeiras é por meio de sementes e, de maneira geral, a germinação é lenta, desuniforme e influenciada por vários fatores relacionados ao ambiente, ou à própria planta, tais como estádio de maturação, presença ou não de pericarpo, tempo entre colheita e semeadura, dormência física, temperatura do ambiente e substrato, entre outros. A germinação, para a maioria das espécies de palmeiras, apresenta frequentemente baixas porcentagens, perdendo a viabilidade rapidamente quando desidratadas (PIVETTA et al., 2007).

Para Luz (2008) e Batista (2009) o estudo da morfologia da semente, bem como da temperatura e do substrato, além de outros fatores que vão atuar no processo germinativo são importantes, pois esses conhecimentos básicos são necessários para que se possam adotar práticas apropriadas para a produção de mudas.

Uma característica marcante em sementes de palmeiras é a variação quanto ao número de dias para germinarem, no entanto, o mecanismo de controle da germinação, de acordo com Carvalho et al. (2005), é pouco conhecido, torna-se necessário adotar algum mecanismo que acelere esse processo (BROSCHAT \& DONSELMAN, 1988).

Nos testes de laboratório, considera-se germinada toda semente que, pela emergência e desenvolvimento das estruturas essenciais do seu embrião, demonstre sua aptidão para produzir planta normal sob condições favoráveis de campo (BATISTA, 2009). Logo, para a obtenção de resultados confiáveis e comparáveis dos testes de germinação, é necessária a utilização de condições padrões, que podem ser encontradas nas Regras para Análises de Sementes.

As espécies nativas, de um modo geral, apresentam crescimento lento, daí a importância de se definir um substrato que promova as velocidades e uniformidades de germinação, aliada à temperatura, ao período adequado de armazenamento e a sementes de boa qualidade fisiológica.

Esta palmeira já é cultivada de forma comercial, no entanto, ainda há poucas pesquisas relacionadas a ela. Sendo que grande parte das informações a respeito da cultura é dada por produtores que, por tentativa, vem procurando melhorar a produtividade e reduzir a idade de corte do palmito Dessa forma, há a necessidade de buscar informações, entre outras, sobre quebra de dormência, espaçamentos, produção de mudas, controle fitossanitário, lançamento de variedades, uniformidade e qualidade do palmito (NASCENTE, 2009).

Neste contexto, percebe-se a necessidade de buscar conhecimentos a respeito de espécies presentes em biomas, onde a pressão antrópica tem se intensificado, como uma tentativa de obter subsídios para elaboração de propostas de conservação e manejo da flora e da fauna. Sob essa ótica, encontram-se as espécies da família Arecaceae, ocorrente nas regiões de cerrado, e que precisam ser mais compreendidas.

Assim, informações sobre fisiologia, anatomia, morfologia, conservação e germinação de sementes são necessárias para o desenvolvimento de tecnologias para produção de sementes e mudas desta palmeira.

Dessa forma, o objetivo do presente trabalho foi verificar se o tamanho do fruto afeta a germinação de sementes provenientes de frutos verdes e maduros de Syagrus oleracea Becc. (Arecaceae).

\section{MATERIAL E MÉTODOS}

O experimento foi desenvolvido no Laboratório de Fisiologia Vegetal e Sementes da Universidade Federal de Goiás (UFG Campus Jataí), latitude de $17^{\circ} 52^{\prime} \quad 53^{\prime \prime} \mathrm{S}$, longitude de $51^{\circ} 42^{\prime} 52^{\prime \prime} \mathrm{W}$ e $672 \mathrm{~m}$ de altitude, no município de Jataí, Goiás, Brasil, no período de agosto de 2009 a junho de 2010.

Os frutos de guariroba foram colhidos manualmente de plantas nativas, nos municípios de Mineiros e Jataí (GO). Após 
isso, foram pesados, individualmente, em balança analítica e mensurados com auxílio de um paquímetro digital, no qual foram obtidas as medidas $(\mathrm{cm})$ de comprimento. Depois de todos os frutos medidos, eles foram separados em cinco classes de tamanho obtidas a partir da diferença entre o maior e o menor comprimento (T1: fruto $\leq 4,300 \mathrm{~cm}$; T2: $4,300 \mathrm{~cm}<$ fruto $\leq 4,833 \mathrm{~cm} ; \mathrm{T} 3: 4,833$ $\mathrm{cm}<$ fruto $\leq 5,367 \mathrm{~cm} ;$ T4: $5,367 \mathrm{~cm}<$ fruto $\leq 5,900 \mathrm{~cm}$ e T5: fruto $>5,900 \mathrm{~cm}$ ).

\section{Germinação}

Para o estudo do processo de germinação foram realizados dois ensaios em épocas diferentes e conduzidos da mesma forma, no entanto, em um experimento foi utilizado frutos verdes (imaturos) e no outro, frutos maduros. Sendo que para os dois ensaios foram utilizados frutos inteiros, constituídos por epicarpo e mesocarpo.

Ensaio 1 - Os frutos verdes (imaturos) foram coletados no mês de julho de 2009 e o experimento foi desenvolvido nos meses de agosto a dezembro de 2009.

Ensaio 2 - Os frutos maduros foram coletados no mês de janeiro de 2010 e o experimento foi desenvolvido nos meses de janeiro a maio de 2010.

Os frutos inteiros, constituídos por epicarpo e mesocaropo, foram semeados em bandejas de plástico e colocados para germinar em Câmara Germinadora tipo BOD, com temperatura constante de $26^{\circ} \mathrm{C}$ e fotoperíodo de 16 horas, em bandejas de plástico $(30 \times 20 \times 10 \mathrm{~cm})$, contendo como substrato o Bioplant, composto por matéria orgânica de origem vegetal e vermiculita inerte.

Em cada ensaio, os experimentos foram montados sob delineamento em blocos casualizados (DBC) com cinco tratamentos (classes de tamanho de frutos) e quatro blocos, contendo 30 frutos cada, totalizando 120 frutos por tratamento e 600 frutos no experimento. Os ensaios foram instalados em duas BOD's, as quais estavam igualmente controladas, sendo que cada uma continha cinco bandejas e, em cada bandeja, constavam duas parcelas (30 frutos por parcela, 60 frutos por bandeja). Os blocos foram distribuídos, usando o gradiente vertical da BOD.

Como os frutos foram colocados para germinar sobre o substrato, foi considerada como germinação a protrusão da radícula, ou seja, $0,5 \mathrm{~cm}$ de comprimento. A avaliação de germinação foi realizada diariamente a partir da protrusão da radícula do primeiro fruto, a fim de que se obtivesse o índice de velocidade de germinação. Essa contagem foi realizada também para se obter o percentual de germinação e o tempo médio gasto para que $50 \%$ do total de frutos germinassem.

Índice de Velocidade de Germinação (IVG): Foi calculado através da metodologia proposta por Maguire (1962) pela expressão:

$$
I V G=\sum\left(\frac{n_{i}}{d_{i}}\right)
$$

Onde:

$n=$ número de frutos germinados na $i$ ésima avaliação;

$d=$ número de dia da $i$-ésima avaliação após o plantio dos frutos.

Porcentagem de Germinação (\%G): Determinou-se a porcentagem de germinação pela expressão proposta nas Regras para Análise de Sementes (BRASIL, 1992):

$$
G \%=\frac{N G \times 100}{N T}
$$

Onde:

$\mathrm{NG}=$ número de frutos germinados

$\mathrm{NT}=$ número total de frutos colocados para germinar

Tempo Médio $\left(\mathbf{T}_{\mathbf{5 0}}\right)$ : Tempo médio, em dias, para atingir $50 \%$ do total germinado, obtido após a estabilização da germinação, verificando a germinação de $50 \%$ dos frutos.

\section{Análises estatísticas}

Foi realizada a análise de variância e teste de Tukey para as variáveis em estudo. Toda a análise foi realizada com auxílio do programa computacional SAEG (2008). 


\section{RESULTADOS E DISCUSSÃO}

Teste de Germinação (Ensaio 1 - Frutos verdes/2009 e Ensaio 2 - Frutos maduros/2010)

Na Tabela 01, estão apresentados os resultados da análise de variância quanto aos valores de número de frutos germinados (NFG), número médio de dias para germinar (NDPG), porcentagem de germinação $(\% \mathrm{G})$, índice de velocidade de germinação (IVG) e tempo médio para germinar $50 \%$ do total germinado $\left(\mathrm{T}_{50}\right)$ de frutos de guariroba.
Pela análise da referida Tabela, observa-se diferenças significativas para número de frutos germinados (NFG), porcentagem de germinação (\%G) e índice de velocidade de germinação (IVG), demonstrando que o tamanho dos frutos de guariroba não exerce influência nas variáveis relacionadas ao tempo (número médio de dias para germinar (NDPG) e tempo médio para germinar metade do total germinado $\left(\mathrm{T}_{50}\right)$ ).

Lin (1986), trabalhando com estádio de maturação e tamanho do fruto de Euterpe edulis, observou que, para os maduros e verdes, o tamanho teve influência na germinação, onde frutos grandes apresentaram maiores médias.

Tabela 01 - Quadrados médios, coeficientes de variação (CV\%) e médias gerais obtidos para caracteres relacionados à germinação de frutos verdes de guariroba (Syagrus oleracea Becc.) em função de diferentes tamanhos de frutos em condições de laboratório

\begin{tabular}{lclllll}
\hline \multirow{2}{*}{ FV } & \multirow{2}{*}{ GL } & \multicolumn{5}{c}{ Quadrado Médio } \\
\cline { 3 - 7 } & & NFG & NDPG & \% G & IVG & T $_{\mathbf{5 0}}$ \\
\hline Tratamento & 4 & $17,13^{* *}$ & 322,99 & $190,28^{* *}$ & $0,007153^{* *}$ & 80,75 \\
Bloco & 3 & 0,98 & 523,36 & 10,93 & 0,0002 & 130,84 \\
Resíduo & 12 & 1,19 & 287,23 & 13,24 & 0,0004 & 71,81 \\
\hline CV(\%) & & 33,59 & 34,44 & 33,59 & 33,04 & 34,44 \\
\hline MÉDIA & & 3,25 & 49,21 & 10,83 & 0,06 & 24,61 \\
\hline
\end{tabular}

**, *: Significativo a 1 e $5 \%$ de probabilidade, respectivamente, pelo teste $\mathrm{F}$

Número de frutos germinados (NFG), Número médio de dias para germinação (NDPG), Porcentagem de germinação (\%G), Índice de velocidade de germinação (IVG) e Tempo médio gasto para germinar metade do total germinado $\left(\mathrm{T}_{50}\right)$

Classes de tamanho: T1: fruto $\leq 4,300 \mathrm{~cm}$; T2: $4,300 \mathrm{~cm}<$ fruto $\leq 4,833 \mathrm{~cm}$; T3: 4,833 cm < fruto $\leq 5,367 \mathrm{~cm} ; \mathrm{T} 4: 5,367$ $\mathrm{cm}<$ fruto $\leq 5,900 \mathrm{~cm}$ e T5: fruto $>5,900 \mathrm{~cm}$

Com relação às médias das variáveis significativas NFG, \% G e IVG, pode-se observar a influência do tamanho do fruto nos tratamentos 2 e 3 , onde foram encontrados os melhores índices quando comparados com os tratamentos 1, 4 e 5 (Tabela 02). 
Tabela 02 - Médias obtidas para caracteres relacionadas à germinação de frutos verdes de guariroba (Syagrus oleracea Becc.) em função de diferentes tamanhos de frutos em condições de laboratório

\begin{tabular}{cllll}
\hline \multirow{2}{*}{ Tratamento } & \multicolumn{5}{c}{ Médias } \\
\cline { 2 - 5 } & NFG & $\mathbf{\% G}$ & IVG & $\mathbf{T}_{\mathbf{5 0}}$ \\
\hline 1 & $2,00 \mathrm{~b}$ & $6,67 \mathrm{~b}$ & $0,0381 \mathrm{~b}$ & $26,46 \mathrm{a}$ \\
2 & $5,50 \mathrm{a}$ & $18,33 \mathrm{a}$ & $0,1080 \mathrm{a}$ & $26,69 \mathrm{a}$ \\
3 & $5,25 \mathrm{a}$ & $17,50 \mathrm{a}$ & $0,1046 \mathrm{a}$ & $25,90 \mathrm{a}$ \\
4 & $2,75 \mathrm{~b}$ & $9,17 \mathrm{~b}$ & $0,0517 \mathrm{~b}$ & $27,36 \mathrm{a}$ \\
5 & $0,75 \mathrm{~b}$ & $2,50 \mathrm{~b}$ & $0,0115 \mathrm{~b}$ & $16,63 \mathrm{a}$ \\
\hline
\end{tabular}

Médias seguidas da mesma letra na coluna não diferem entre si pelo teste de Tukey a $5 \%$ de probabilidade

Número de frutos germinados (NFG), Número médio de dias para germinação (NDPG), Porcentagem de germinação (\%G), Índice de velocidade de germinação (IVG) e Tempo médio gasto para germinar metade do total germinado $\left(\mathrm{T}_{50}\right)$

Classes de tamanho: T1: fruto $\leq 4,300 \mathrm{~cm}$; T2: 4,300 cm < fruto $\leq 4,833 \mathrm{~cm}$; T3: 4,833 cm < fruto $\leq 5,367 \mathrm{~cm} ; \mathrm{T} 4: 5,367$ $\mathrm{cm}<$ fruto $\leq 5,900 \mathrm{~cm}$ e T5: fruto $>5,900 \mathrm{~cm}$

Na Tabela 03, estão apresentados os resultados da análise de variância para os frutos maduros. Quanto aos valores do número de frutos germinados (NFG), percentual de germinação $(\% \mathrm{G})$, índice de velocidade de germinação (IVG), número médio de dias para germinar (NDPG) e tempo para germinar $50 \%$ do que germinou $\left(\mathrm{T}_{50}\right)$ de frutos de guariroba.

Pela análise da referida Tabela, observa-se que para NDPG, IVG e $\mathrm{T}_{50}$, foram constatadas diferenças significativas entre os diferentes tamanhos de frutos estudados. E que para NFG e \%G não foram constatadas diferenças significativas entre os tratamentos, indicando que o tamanho de frutos afeta a velocidade de germinação e não a quantidade de frutos germinados no caso da guariroba.

A Tabela 04 demonstra que frutos do tratamento 3 necessitam de menor período de tempo para germinarem NDPG (42,07 dias) e IVG $(0,5128)$ com processo germinativo mais rápido $\mathrm{T}_{50}$ (21,04 dias). No entanto, frutos do tratamento 4 necessitam de maior período de tempo para germinarem NDPG $(58,41$ dias $)$ e IVG $(0,2522)$ com processo germinativo mais lento $T_{50} \quad(29,21$ dias $)$. Esses resultados evidenciam que frutos maduros com tamanho entre 4,834 e 5,367 cm germinam em menor espaço de tempo, e que frutos maduros com tamanho entre 5,368 e $5,900 \mathrm{~cm}$ necessitam mais tempo para germinarem.

Embora não tenha sido ressaltada diferença significativa no percentual de germinação, pela análise das médias pode-se verificar que os frutos do tratamento 3 possuem maior valor $(67,50 \%)$ e frutos do tratamento 4 possuem menor valor $(44,17 \%)$. 
Tabela 03 - Quadrados médios, coeficientes de variação (CV\%) e médias gerais obtidos para caracteres relacionadas à germinação de frutos maduros de guariroba (Syagrus oleracea Becc.) em função de diferentes tamanhos de frutos em condições de laboratório

\begin{tabular}{lllllll}
\hline \multirow{2}{*}{ FV } & \multirow{2}{*}{ GL } & \multicolumn{5}{c}{ Quadrado Médio } \\
\cline { 3 - 7 } & & NFG & NDPG & \%G & IVG & T $_{\mathbf{5 0}}$ \\
\hline Tratamento & 4 & 26,38 & $158,71^{* * *}$ & 293,06 & $0,0377^{* * *}$ & $39,68^{* *}$ \\
Bloco & 3 & 3,65 & 10,60 & 40,56 & 0,0014 & 2,65 \\
Resíduo & 12 & 9,61 & 18,41 & 106,76 & 0,0048 & 4,60 \\
\hline CV(\%) & & 19,08 & 8,61 & 19,08 & 18,56 & 8,61 \\
\hline MÉDIA & & 16,25 & 49,86 & 54,17 & 0,3730 & 24,93 \\
\hline
\end{tabular}

*, **: Significativo a 1 e $5 \%$ de probabilidade, respectivamente, pelo teste $\mathrm{F}$

Número de frutos germinados (NFG), Número médio de dias para germinação (NDPG), Porcentagem de germinação (\%G), Índice de velocidade de germinação (IVG) e Tempo médio gasto para germinar metade do total germinado $\left(\mathrm{T}_{50}\right)$

Classes de tamanho: T1: fruto $\leq 4,300 \mathrm{~cm}$; T2: 4,300 cm < fruto $\leq 4,833 \mathrm{~cm}$; T3: 4,833 cm < fruto $\leq 5,367 \mathrm{~cm}$; T4: 5,367 $\mathrm{cm}<$ fruto $\leq 5,900 \mathrm{~cm}$ e T5: fruto $>5,900 \mathrm{~cm}$

Tabela 04 - Médias obtidas para caracteres relacionadas à germinação de frutos maduros de guariroba (Syagrus oleracea Becc.) em função de diferentes tamanhos de frutos em condições de laboratório

\begin{tabular}{clll}
\hline \multirow{2}{*}{ Tratamento } & \multicolumn{3}{c}{ Médias } \\
\cline { 2 - 4 } & NDPG & IVG & T $_{\mathbf{5 0}}$ \\
\hline 1 & $51,12 \mathrm{abc}$ & $0,3672 \mathrm{ab}$ & $25,56 \mathrm{abc}$ \\
2 & $52,10 \mathrm{ab}$ & $0,3254 \mathrm{~b}$ & $26,05 \mathrm{ab}$ \\
3 & $42,07 \mathrm{c}$ & $0,5128 \mathrm{a}$ & $21,04 \mathrm{c}$ \\
4 & $58,41 \mathrm{a}$ & $0,2522 \mathrm{~b}$ & $29,21 \mathrm{a}$ \\
5 & $45,58 \mathrm{bc}$ & $0,4075 \mathrm{ab}$ & $22,79 \mathrm{bc}$ \\
\hline
\end{tabular}

Médias seguidas da mesma letra na coluna não diferem entre si pelo teste de Tukey a $5 \%$ de probabilidade

Classes de tamanho: T1: fruto $\leq 4,300 \mathrm{~cm}$; T2: 4,300 cm $<$ fruto $\leq 4,833 \mathrm{~cm}$; T3: 4,833 cm $<$ fruto $\leq 5,367 \mathrm{~cm}$; T4: 5,367

$\mathrm{cm}<$ fruto $\leq 5,900 \mathrm{~cm}$ e T5: fruto $>5,900 \mathrm{~cm}$

Com relação à comparação entre frutos verdes e maduros (Tabelas 02 e 04 respectivamente), observa-se que os frutos maduros têm maior capacidade de germinação. Os frutos maduros possuem, em média, cinco vezes mais capacidade de germinação e seis vezes mais velocidade de germinação que os frutos verdes. Entretanto, o tempo médio para germinar $50 \%$ do total de germinação e o número médio de dias para germinar, se apresentaram bem próximos, sendo $\mathrm{T}_{50}: 24,93$ e 24,61, e NDPG: 49,86 e 49,21, para frutos maduros e verdes, respectivamente. Dentre outras espécies, a Syagrus romanzoffiana (jerivá) apresenta, ao contrário da Syagrus oleracea Becc., maior capacidade germinativa para frutos verdes (BROSCHAT \& DONSELMAN, 1988) e Viana (2003), também obteve melhores resultados em sementes obtidas de frutos verdes de Livistona rotundifolia. Já para as espécies Roystonea regia (PENARIOL, 2007) e Euterpe edullis (ANDRADE et al., 1996) a germinação também apresentou melhores resultados com frutos maduros da mesma forma que acontece com a guariroba (Syagrus oleracea Becc.).

\section{CONCLUSÕES}

Há maiores e menores taxas de germinação, respectivamente, para frutos que possuem comprimento entre 4,834 e $5,367 \mathrm{~cm}$ (Tratamento 3) e entre 5,368 e $5,900 \mathrm{~cm}$ (Tratamento 4) em condições de laboratório, e 
Frutos maduros possuem maior capacidade de germinação quando comparados com frutos verdes.

\section{REFERÊNCIAS}

ALVES, E. U.; BRUNO, R. L. A.; OLIVEIRA, A. P.; ALVES, A. U.; ALVES, A. U.; PAULA, R. C. Influência do tamanho e da procedência de sementes de Mimosa caesalpiniifolia Benth. sobre a germinação e vigor. Revista Árvore, Viçosa, v.29, n.6, p.877-885. 2005.

ANDRADE, A. C. S. de; VENTURI, S.; PAULINO, M. T. S. Efeito do tamanho das sementes de Euterpe edulis Mart. sobre a emergência e crescimento inicial. Revista Brasileira de Sementes, v.18, n.2, p, 225231. 1996.

BATISTA, G. S. Morfologia e germinação de sementes de Syagrus oleracea Becc. (Mart.) Becc (Arecaceae). 2009. 46f. Dissertação (Mestrado em Agronomia) Faculdade de Ciências Agrárias e Veterinárias, Universidade Estadual Paulista, Jaboticabal, 2009.

BRASIL. Ministério da Agricultura. Regras para Análise de Sementes. Brasília: Secretaria Nacional de Defesa da Agropecuária, 365 p. 1992.

BROSCHAT, T. K.; DONSELMAN, H. Palm seeds torage and germination studies. Principes, Lawrence, v. 32, n. 1, p. 3-12. 1988.

CARVALHO, N. M.; NAKAGAWA, J. Germinação de sementes. In: CARVALHO, N. M.; NAKAGAWA, J. Sementes: ciência, tecnologia e produção. 4. ed. Jaboticabal: FUNEP, 2000, p.128-166.

CARVALHO, N. O. S.; PELACANI, C.R.; RODRIGUES, M. O. de. S.; CREPALDI, I. C. Uso de substâncias reguladoras e nãoespecíficas na germinação de sementes de licuri (Syagrus coronata (MART.) BECC). Sitientibus Série Ciências Biológicas v.5, n.1, p.28-32. 2005.
GENTIL, D. F. O.; FERREIRA, S.A.N. Morfologia da plântula em desenvolvimento de Astrocaryum aculeatum Meyer (Arecaceae). Acta Amazonica, Manaus, v.35, n.3, p.337-342. 2005.

IOSSI, E. Morfologia floral e maturação fisiológica de sementes de tamareira-anã (Phoenix roebelenii O'Brien) - Arecaceae. 2005. 63f. Tese (Doutorado em Agronomia) Faculdade de Ciências Agrárias e Veterinárias, Universidade Estadual Paulista, Jaboticabal, 2005.

LIN S. S. Efeito do tamanho e maturidade sobre a viabilidade, germinação e vigor do fruto de palmiteiro. Revista Brasileira de Sementes, v.8, n.1, p.57-66. 1986.

LORENZI, H. Palmeiras brasileiras e exóticas cultivadas. Nova Odessa: Plantarum, 416p. 2004.

LUZ, P. B. Germinação e aspectos morfológicos de sementes de Archontophoenix cunninghamii $\mathbf{H}$. Wendl. \& Drude (Arecaceae). 2008. 63f. Tese (Doutorado em Agronomia) - Faculdade de Ciências Agrárias e Veterinárias, Universidade Estadual Paulista, Jaboticabal, 2008.

MAGUIRE, J. D. Speed of germination - aid in selection and evaluation for seedling emergence and vigor. Crop Science, Madison, v.2, n.2, p.176-177. 1962.

NASCENTE, A.S. Guariroba (Syagrus oleracea Becc. Becc) - o palmito do cerrado. Disponível em: http://www.fazendeiro.com.br/CieTec/artigos /ArtigosTexto.asp?Codigo=474. Acesso em: 06 de março de 2009.

PENARIOL, A. P. Germinação e Morfologia de Sementes de Roystonea regia (Kunth) O. F. Cook. (Arecaceae). 2007. 40f. Dissertação (Mestrado em Agronomia) Faculdade de Ciências Agrárias e Veterinárias, Universidade Estadual Paulista, Jaboticabal, 2007.

PIVETTA, K. F. L.; BARBOSA, J. G., ARAÚJO, E. F. Propagação de palmeiras e strelitzia. In: BARBOSA, J. G.; LOPES, L. 


\section{Propagação de Plantas Ornamentais.}

Viçosa: UFV, 2007, p. 43-70.

SAEG - Sistema para Análise Estatística e Genética. Viçosa: UFV-Funarbe, 2008.

SANTELLI, P.; CALBO, M. E. R.; CALBO, A. G. Fisiologia Pós-Colheita de Frutos da Palmeira Syagrus oleracea Becc.(Mart.) Becc. (Arecaceae). Acta Botânica Brasileira, v. 20, n. 3, p. 523-528. 2006.

VIANA, F. A. P. Estudos sobre a germinação e morfo-anatomia do diásporo e da plântula de Livistona rotundifolia (Lam.) Mart. (Arecaceae). 2003. 76f. Dissertação (mestrado em Agronomia) Faculdade de Ciências Agrárias e Veterinárias, Universidade Estadual Paulista, Jaboticabal, 2003. 